\title{
Subsurface morphology changes due to deuterium bombardment of tungsten
}

\author{
S. Lindig ${ }^{\text {a, }}$, M. Balden ${ }^{\mathrm{a}}$, V.Kh. Alimov ${ }^{\mathrm{b}}$, T. Yamanishi ${ }^{\mathrm{b}}$, W.M. Shu ${ }^{\mathrm{c}}$, J. Roth $^{\mathrm{a}}$ \\ ${ }^{a}$ Max-Planck-Institut für Plasmaphysik, EURATOM Association, D-85748 Garching, \\ Germany \\ ${ }^{b}$ Tritium Technology Group, Japan Atomic Energy Agency, Tokai, Ibaraki, 319-1195, Japan \\ ${ }^{c}$ ITER International Organization, Cadarache, 13108 Saint Paul-lez-Durance, France
}

\begin{abstract}
Re-crystallized polycrystalline tungsten was exposed to a deuterium plasma beam with high flux $\left(10^{22} \mathrm{D} / \mathrm{m}^{2} \mathrm{~s}\right)$ and low energy (38 eV/D) to fluences up to $10^{27} \mathrm{D} / \mathrm{m}^{2}$. The sample temperature was varied between $320 \mathrm{~K}$ and $800 \mathrm{~K}$. The three-dimensional morphology of blister-like structures and the grain orientation were investigated by scanning electron microscopy combined with focused ion beam cutting and electron backscattering diffraction. Cracks with distorted areas $(<650 \mathrm{~K})$ and large cavities $\left(100-1000 \mu^{3}\right)$ at grain boundaries $(>480 \mathrm{~K})$ were observed beneath the surface. The surface blister-like structures and the defects underneath are correlated along crystallographic orientation of the $W$ grains in accordance to the low-indexed slip system $\{110\}<111>$. The defects are mobile and accumulate under deuterium-loading. Samples exposed near room temperature do not form such large cavities by subsequent heating up to $1300 \mathrm{~K}$. Deuterium bombardment above $700 \mathrm{~K}$ does not lead to blister-like structures.
\end{abstract}

PACS numbers: 52.40.Hf, 61.72.Qq, 61.80.Jh

\section{Introduction}

The assessment of the tritium inventory in future thermonuclear fusion devices and, therefore, the retention of hydrogen isotopes in plasma-facing material, e.g. tungsten, are of major interest [1]. Most of the results on hydrogen isotope retention and recycling for $\mathrm{W}$ have been reviewed $[2,3,4]$. Deep inward diffusion and trapping of $\mathrm{D}$ far beyond the ion range is reported. Quite often the D retention is accompanied by formation of blister-like surface structures ([4] and references therein, $[5,6,7,8,9,10,11])$, which is assumed to be responsible for a minimum in the deuterium depth profiles [12].

Such morphological changes due to ion-loading of tungsten could lead to additional material loss, e.g., by exfoliation, overheating of the thermally poorly connected structures, and bursting of the blister cap with release of $\mathrm{D}_{2}$ and $\mathrm{He}[5,13]$. The three-dimensional (3D) morphology of the defects has to be known to assess such additional material release and the consequences for the overall behaviour in fusion devices, e.g., production of dust, pollution of plasma, and lifetime of plasma-facing components.

In the literature many research groups report on the formation of blisters-like structures on tungsten by hydrogen isotope and helium impact $[5,6,7,8,9,10,12,13,14,15,16,17,18,19$, 20,21]. Most publications describe SEM investigations of surface features and only a few investigations deal with their subsurface morphology $[7,8,11,17,18,19]$. The observed shape, size, and number of the blister-like structures depend strongly on the exact exposure 
conditions, like ion species (e.g. impurities [12,15]), impact energy, flux, fluence, and temperature $[6,7,8,9,10,15,22,23]$. Beyond that, the material properties of the tungsten material have impact on the morphology of the blister-like structures, e.g., grain size and texture, impurities, and defect densities (vacancies, voids, and dislocations). Special care has to be devoted on the thermal history of the specimen as well as on the surface finishing, e.g., mechanical and electrochemical polishing. All this has to be taken into account for comparing between different studies.

In this study, equivalent specimens of re-crystallized tungsten were exposed to a low energy (38 eV/D), high flux $\left(10^{22} \mathrm{D} / \mathrm{m}^{2} \mathrm{~s}\right)$ deuterium plasma beam up to $10^{27} \mathrm{D} / \mathrm{m}^{2}$ at various temperatures from near room temperature (RT) up to $800 \mathrm{~K}$. These $\mathrm{W}$ specimens belong to a measurement series, in which the dependence of deuterium retention on specimen temperature, fluence, and the addition of $\mathrm{He}$ ions into the $\mathrm{D}$ plasma beam has been determined [22]. The mixed bombardment simulates more realistically the situation of a burning fusion plasma, e.g. in the ITER divertor.

The deuterium retention has been analysed using nuclear reaction analysis (NRA) and thermal desorption spectroscopy (TDS) [22,23]. At a fluence of $10^{26} \mathrm{D} / \mathrm{m}^{2}$ the temperature dependence of the D retention shows a quite narrow maximum of nearly $10^{22} \mathrm{D} / \mathrm{m}^{2}$ slightly above $500 \mathrm{~K}$, while at about RT only $\sim 2 \times 10^{20} \mathrm{D} / \mathrm{m}^{2}$ is retained. Above $650 \mathrm{~K}$, the retained amount drops below RT level. For the ten times higher fluence $\left(10^{27} \mathrm{D} / \mathrm{m}^{2}\right)$, the maximum shifts to slightly lower temperature $(<500 \mathrm{~K})$ and the retained amount increases slightly above $10^{22} \mathrm{D} / \mathrm{m}^{2}$. At temperatures above $650 \mathrm{~K}$, no differences in the retention have been observed for both fluences, while for the retention below $500 \mathrm{~K}$ a strong increase has been found, e.g., at RT to above $10^{21} \mathrm{D} / \mathrm{m}^{2}[22,23]$. It has to be noted that such a maximum in the $\mathrm{D}$ retention has been reported for different experimental condition $[6,12,24,25]$. The dependency on fluence was studied in a previous investigation. There it was shown that the content of retained D increases with square root of fluence, which points to a diffusion driven mechanism [14].

As it was shown recently, an appearance of two-types of blister-like structures, small ones (around $1 \mu \mathrm{m})$ and large ones $(\sim 10 \mu \mathrm{m})$, as a function of the irradiation temperature, coexists around the maximum in the $\mathrm{D}$ retention $[8,14]$. Their morphology contradicts the typical blister definition as described by Scherzer with thin cap thickness compared to the lateral dimension of the circular blisters [13]. Nevertheless, the expression 'blister' is used to describe these surface structures induced by D bombardment.

This paper focuses on the variation of the subsurface morphology depending on temperature and the implication for the formation mechanisms analyzed by scanning electron microscopy (SEM). The unique possibilities of position-selective, sub-micrometer precise crosssectioning with a focused ion beam (FIB) is employed for cutting through the blister-like structure. In addition, electron backscattering diffraction (EBSD) is used to determine the orientation of the individual grains.

\section{Experimental}

Mechanically and electrochemically polished specimens of polycrystalline tungsten, purity of $99.99 \mathrm{wt} \%$ (A.L.M.T. Corp., Japan), were re-crystallized at $2073 \mathrm{~K}$ for $1 \mathrm{~h}$. The resulting typical grain size was $20-100 \mu \mathrm{m}$. The specimens were exposed to a deuterium plasma beam from a linear plasma generator [26]. The plasma beam consists mainly of $\mathrm{D}_{2}{ }^{+}$, over $80 \%$. The main impurity in the plasma was oxygen with a concentration less than $1 \mathrm{ppm}$ [26]. The impact energy per $\mathrm{D}$ atom was chosen to $38 \mathrm{eV}$ by applying a bias voltage to the $\mathrm{W}$ specimen $(-80 \mathrm{~V})$ and knowledge of the plasma potential (about $-4 \mathrm{~V}$, measured by a Langmuir probe). 
The incident deuterium flux was $10^{22} \mathrm{D} / \mathrm{m}^{2} \mathrm{~s}$. The accumulated fluence on the investigated specimens was $10^{26} \mathrm{D} / \mathrm{m}^{2}$ or $10^{27} \mathrm{D} / \mathrm{m}^{2}$.

The main parameter changed in the experimental series was the temperature during plasma exposure, which was varied between $320 \mathrm{~K}$ and $800 \mathrm{~K}$. The temperature of the specimen was measured with a thermocouple pressed onto the backside of the specimen [25]. The temperature was adjusted by varying the thermal contact of the specimen to the target holder in order to change the effective cooling of the plasma-heated specimen.

The D retention of these specimens was determined by NRA and TDS. The results are presented separately $[22,23]$.

The surface topography and the 3D morphology of the deuterium-exposed tungsten were analyzed by SEM. The 3D information was obtained by sequential cross-sectioning with a FIB and imaging (SEM). The used device, HELIOS NanoLab 600, FEI, combines both beams and allows automated sequencing of cross-sectioning and imaging. Because the distance between each image is precisely controlled, the $3 \mathrm{D}$ structures can be evaluated and displayed as a movie. The angle between the ion and electron beam is $52^{\circ}$. The surface normal was aligned to the ion beam for the cross-sectioning. The Ga ions had an impacting energy of 30 $\mathrm{keV}$ and the current of the ion beam was varied between 1-25 nA. The SEM images were typically taken using a $5 \mathrm{keV}$ electron beam. To reduced artefacts of the cutting process (e.g. curtaining effect), the area of interest was coated in-situ with a Pt-C film prior to the cutting. The introduced precursor gas $\left(\mathrm{C}_{9} \mathrm{H}_{16} \mathrm{Pt}\right)$ was cracked locally by scanning one of the two beams over a selected area.

In addition, the observed blister-like structures are correlated to the crystallographic orientation of the individual grains. The grain orientation was determined by EBSD. A $20 \mathrm{keV}$ electron beam with an incident angle of $70^{\circ}$ to the normal of the analysed surface area was typically used for the EBSD analysis with the NordlysII-detector, HKL, and channel 5 analysis software, HKL.

\section{Results}

Figures 1 and 2 show examples of the re-crystallized tungsten surface exposed to $38 \mathrm{eV} / \mathrm{D}$ to a fluence of $10^{27} \mathrm{D} / \mathrm{m}^{2}$ at different temperatures. For comparison, in Fig. 3 the surface and a cross-section of virgin specimen are presented. At $320 \mathrm{~K}$, only sparse flat blisters appear of irregular shape and of a size of ca. $1-5 \mu \mathrm{m}$. At $480 \mathrm{~K}$, the density of blisters is more than an order of magnitude higher than at $320 \mathrm{~K}$, by far the most of the blisters have a size of $0.5-5$ $\mu \mathrm{m}$ and only a few larger blisters appear with dimensions of 5-30 $\mu \mathrm{m}$. The ratio height to lateral size for the small ones reaches about 1 . The number of the larger blisters increases with increasing temperature and these blisters show often a few terraces with flat parts parallel to the surface (Fig. 2). At $600 \mathrm{~K}$, still small blisters are present, sometimes even on top of larger blisters (Fig. 2), but their density is less than at temperatures of 450-550 K. In this temperature range, the highest density of structural change is observed. A few exceptional large blisters with a lateral dimension of $>100 \mu \mathrm{m}$ are observed, but their range is always inside of one grain. They do not cross grain boundaries. At higher temperatures $(\geq 700 \mathrm{~K})$, there are no visible changes on the surface and subsurface. They look like the virgin surfaces (Fig. 3).

Only a part of the large variety of observed blister shapes is shown in Figs. 1 and 2. Also for some grains obviously, shape and position of the blister are correlated to the grain orientation, which was determined by EBSD (Fig. 4). The elongation direction of the blisters coincides with the projection of the $\{110\}$ high symmetry plans of the W crystals onto the surface. 
The cross-section of the large blisters at elevated temperatures always exhibits a cavity deep beneath the surface, which has contact with a grain boundary (Fig. 2b). The thickness of the material above the cavity reaches easily $10 \mu \mathrm{m}$, some of the cavities extend to even more than $50 \mu \mathrm{m}$ beneath the surface. The volume of these cavities is roughly equal to the volume of the blister above the surface and is typically in the range of $100-1000 \mu \mathrm{m}^{3}$. The shape of the surface topography is a transformed image of the cavity shape. The cavities are often not exactly below the blisters. The lateral shift is correlated to the grain orientation.

The cross-section of the small blisters is correlated at all temperatures with crack-like structures beneath the surface (several $\mu \mathrm{m}$ ), which sometimes open up to pores. Fig. $1 \mathrm{~b}$ gives an impressive example of this situation, but also in the Figs. 1a and $2 b$ these structures are visible below the small blisters. Generally, the occurrence of these structures depends strongly on the temperature. A possible dependence on the grain orientation is obscured by the variation already inside of single grains. In addition, sometimes cracks deeper along the grain boundaries appear, even below the second grain under the surface (Fig. 1b).

Fig. 1b highlights another important feature of the D-exposed W surface: large distorted regions around the cracks, visible as different greyscales inside a grain. (Due to the channelling contrast of the backscattered electrons, areas of equal crystallographic orientation, i.e. grains, appear with the same greyscale.) Such distorted subsurface regions are in a few cases present without any significant indication on the surface.

From investigating specimens before and after the TDS heating [22,23], it can be concluded that subsequent heating up to $1300 \mathrm{~K}$ after implantation (e.g. during TDS experiments) does not change there morphology. The distorted regions do not anneal, cracks and large cavities in specimens are not formed.

\section{Discussion}

The observation of cracks, distorted areas, and large cavities beneath the surface on Dimplanted $\mathrm{W}$ indicates that the stress during D implantation exceeds the toughness of the material due to a $\mathrm{D}$ concentration exceeding the solubility $[4,5,8,12]$. The weak points in the material could be the defects, like vacancies, voids, dislocations, and grain boundaries, on which the deuterium can agglomerate $[27,28]$.

At elevated temperatures, by overcoming the brittle-to-ductile-transition (BDT) $[29,30]$, the stress can be relaxed by dislocations moving along lattice planes though the whole crystallite leading to the cavities at the grain boundaries, because the dislocation mobility is increased by this transition. This corresponds to material migration above the surface, i.e., the blister-like surface topography. However, near RT the stress relaxation results in plastic deformation and crack formation inside the grains. The coexistence of all features is in line with the dependence of the BDT temperature on various parameters, like vacancies and dislocation density and loading rate [29,30].

The location of the cavity in respect to the surface structure as well as the direction of the elongation of the blister-like structures (Figs. 2 and 4) can be explained by the gliding in the known low-indexed slip system $\{110\}<111>$. This means gliding along the $<111>$ direction in the $\{110\}$ planes. To verify if higher-indexed slip systems are also active, as discussed in the literature $[31,32]$, further detailed investigations are necessary.

The co-incidence of the maximum of the $\mathrm{D}$ retention in the temperature dependency [22,23] with the appearance of highest density of structural changes is evident. But it has to be stressed that the retained D after the implantation, which is determined by TDS and NRA $[22,23]$, does not describe the D lattice concentration during the implantation. The retained D 
measured many days after the implantation relates mainly to the trap density and the gas-filled pore volume $[33,34]$. But the transient $\mathrm{D}$ concentration during implantation is responsible for the stress state. An indication for that is also that the retained D together with annealing does not form the large cavities as it does during implantation. This implies that the mobility of vacancies, voids, and dislocations in tungsten is affected by the transient $\mathrm{D}$ inventory during the implantation and the implied stress.

The absence of blister-like structures as well as the low D retention at temperatures above $700 \mathrm{~K}$ indicates that the requirement for extended defect production is not fulfilled. Possibly, it could be explained by the strongly increased solubility and diffusivity [2] avoiding that the transient D concentration exceeds the solubility. Also it could be speculated that the defects in $\mathrm{W}$ anneal at these temperatures, the reemission is increased, and therefore, the transient $\mathrm{D}$ concentration is strongly lowered. Note that the grain boundaries hinder the D diffusion and, therefore, the full 3D morphology of the $\mathrm{W}$ material determines the transient $\mathrm{D}$ concentration.

\section{Summary}

Re-crystallized polycrystalline tungsten was exposed to a deuterium plasma with high flux of $10^{22} \mathrm{D} / \mathrm{m}^{2} \mathrm{~s}$ of $\sim 38 \mathrm{eV} / \mathrm{D}$ up to fluences of $10^{26}$ and $10^{27} \mathrm{D} / \mathrm{m}^{2}$ and temperature between $320 \mathrm{~K}$ and $800 \mathrm{~K}$. The observed surface topography varies with temperature, fluence and depends strongly on the grain orientation. By cross-sectioning with a FIB through the blister-like structures on the surface, their subsurface morphology and position is accessible and analysed using SEM.

With increasing temperature, the observable cracks below the surface increase in length, number and width. At $\sim 360 \mathrm{~K}$ the fine crack-like defects inside grains show a length $\sim 5 \mu \mathrm{m}$ and width: $\sim 0.1 \mu \mathrm{m}$ up to a depth of $\sim 10 \mu \mathrm{m}$. Around the cracks often large distorted areas are observed. At higher temperatures $(500 \mathrm{~K}-650 \mathrm{~K})$, large cavities at the grain boundaries together with their large surface structures are the dominating features and the fine-crack structures with the distorted region around loose their significance. For temperatures $\geq 700 \mathrm{~K}$, no blister-like structures are detected.

In most cases a correlation of shape and position of the defect structures underneath a surface structure with the crystallite orientation is obvious. Especially this is shown at temperatures between $500 \mathrm{~K}$ and $650 \mathrm{~K}$ for the large cavities at the grain boundaries. Probably also at lower temperatures $(<500 \mathrm{~K})$ the cracks show orientation dependence, but which are not sufficiently studied at this time. An explanation could be that tungsten behaves brittle at temperatures less than $\sim 500 \mathrm{~K}$ and the stress by the high transient D inventory during implantation deforms plastically the grains in the distorted area and creates cracks within the grains. At higher temperatures (until $650 \mathrm{~K}$ ) the brittle to ductile transition, which is dependent on stress and on material parameters like the solved hydrogen content, causes that cracks or deformation of crystal planes do not appear within the grains. But large cavities arise only on the grain boundaries. Material can be moved out easily along gliding planes and in corresponding direction in the low-index slip system $\{110\}<111>$ to reduce mechanical stress by transient D inventory. The movement is supported probably by $\mathrm{D}$ gas pressure in the cavity from the super-saturated bulk inventory. Therefore the surface structures are elongated along the gliding plane and the position relative to the cavity is determined by the slip system direction. This process forms surfaces structures which reflect the shape of the cavity. Post-irradiation annealing up to $1300 \mathrm{~K}$ does not form the large cavities. This implies that the mobility of dislocations in tungsten is affected by the high transient $\mathrm{D}$ inventory during the implantation and not by temperature or gas pressure effects during post-irradiated heat treatment. 


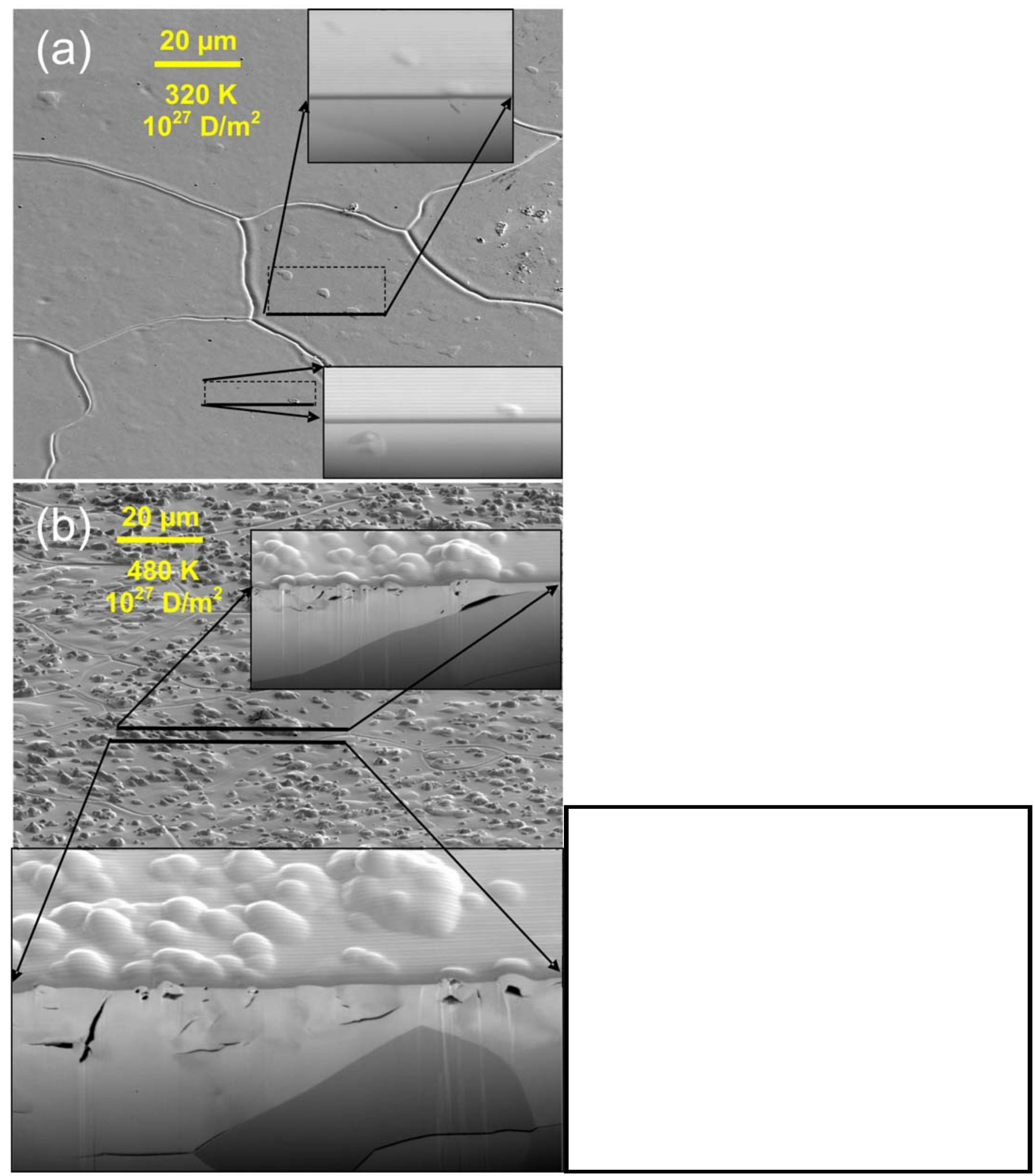

Fig. 1: Tungsten surface exposed to $10^{27} \mathrm{D} / \mathrm{m}^{2}$ at $320 \mathrm{~K}$, surface tilted by $52^{\circ}$ (a) and at $480 \mathrm{~K}$, tilted by $70^{\circ}$ (b). The inserts in (a) show the cross-section through one of the sparse blisters as well as through a distorted region below the surface, which does not exhibit a noticeable blister at the surface. Inserts in (b) show strongly distorted and cracked regions, the upper overlay show also a cavity at grain boundary beneath the large blisterlike structure. The bolt lines indicate the positions of the cross-sections with marked surface area shown in inserts (all inserts tilted by $52^{\circ}$ ). Surface in inserts was coated by Pt-C-film. Additional to (b) a movie of progressive cross-sectioning is available on the web system of publisher. 

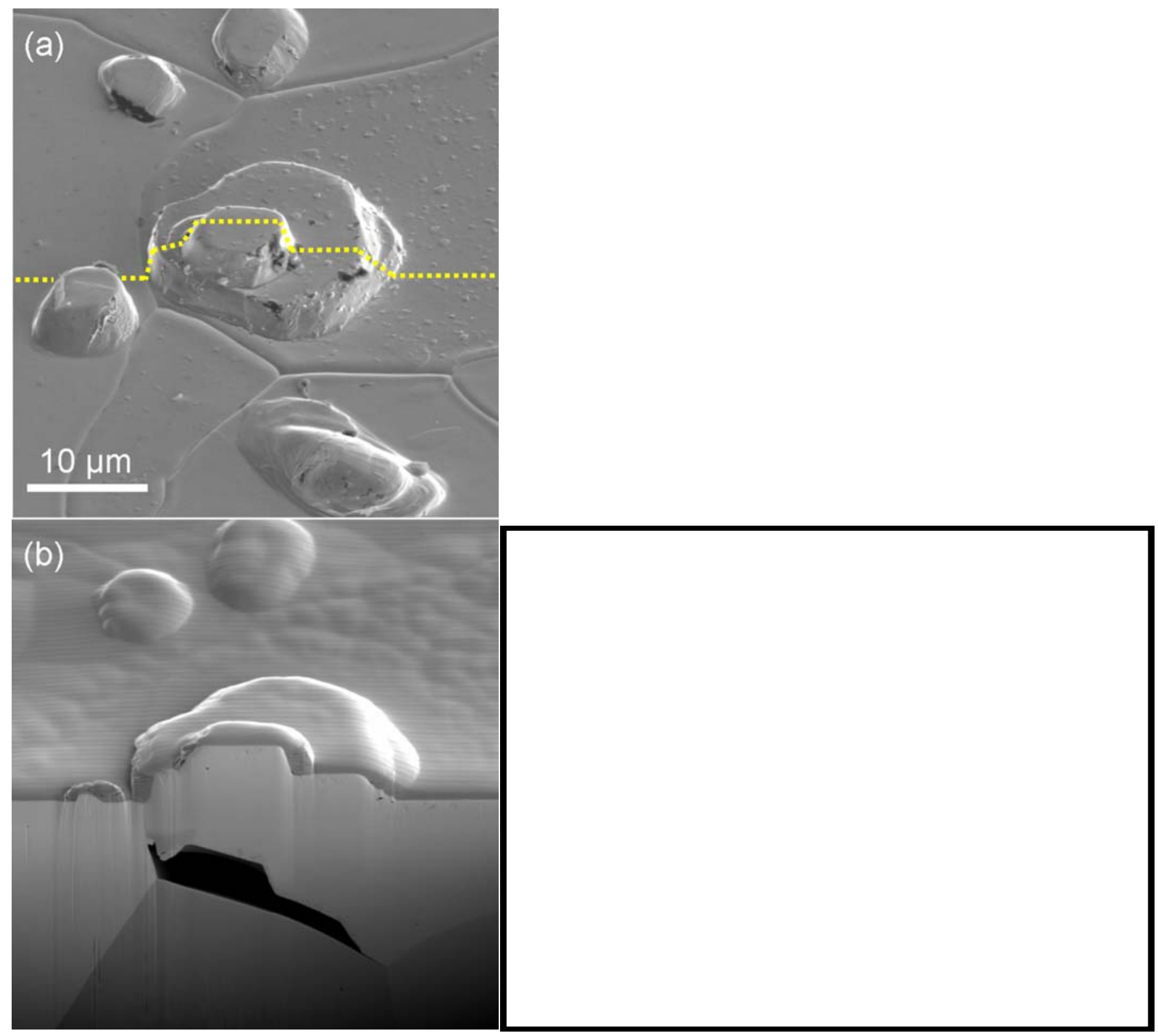

Fig 2: Tungsten surface exposed to $10^{27} \mathrm{D} / \mathrm{m}^{2}$ at $600 \mathrm{~K}$ with stepped high-dome blister (a) before and (b) after cross-sectioning with FIB (surface tilted by $52^{\circ}$; cross-section by $-38^{\circ}$ ).

Line in (a) indicates the position of cross-sectioning (b). The surface in (b) is coated with a mixed Pt-C film. Additional a movie of progressive cross-sectioning is available on the web system of publisher. 


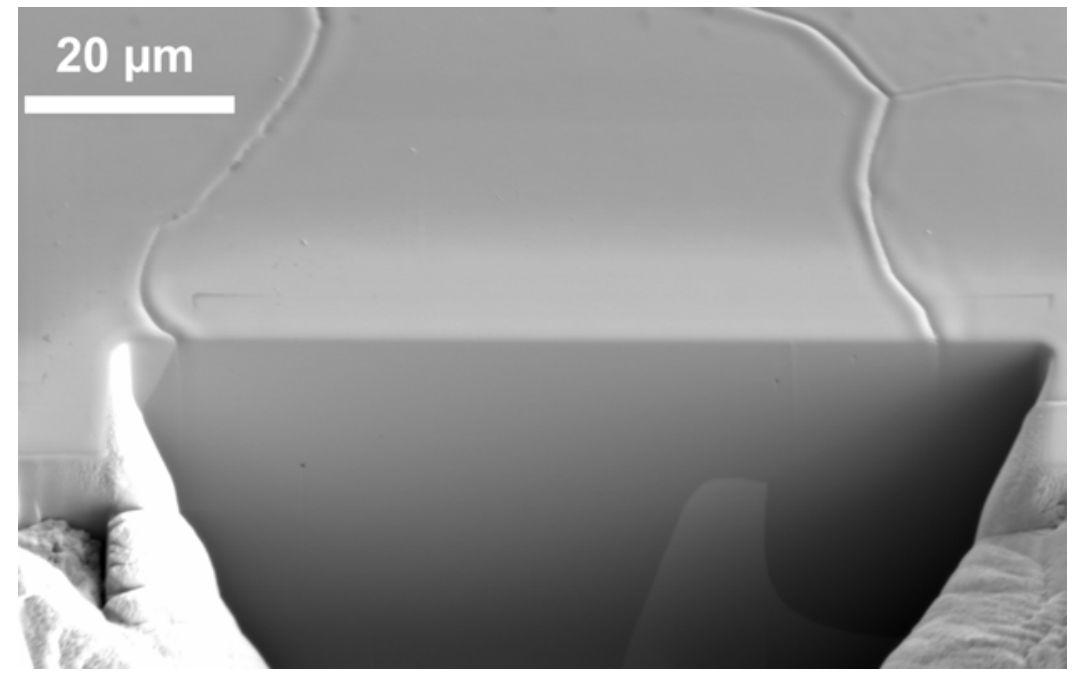

Fig 3: SEM image of a virgin tungsten specimen. In the lower part the subsurface is made visible by crosssectioning with FIB.

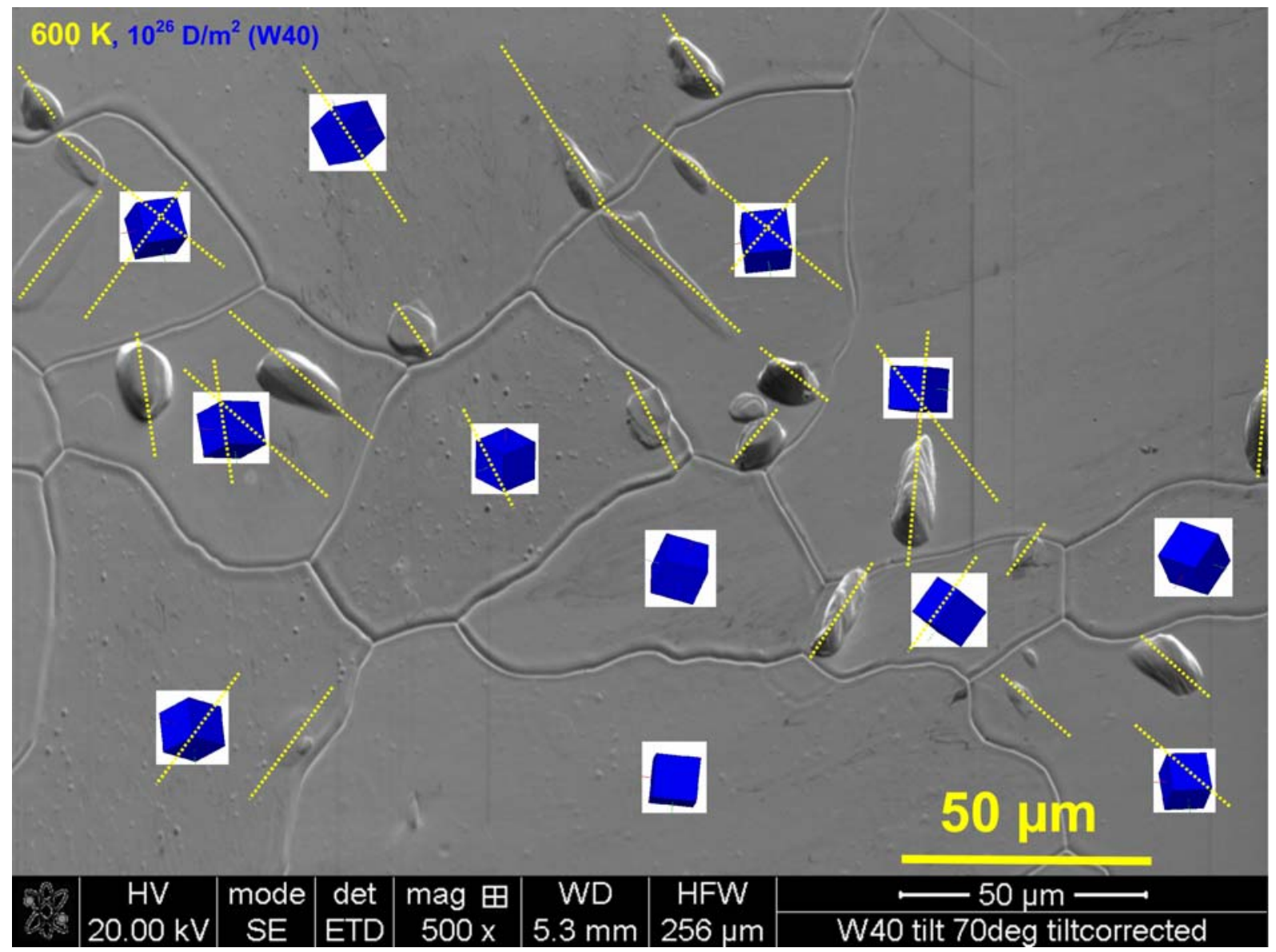

Fig 4: Tungsten surface exposed to $10^{26} \mathrm{D} / \mathrm{m}^{2}$ at $600 \mathrm{~K}$ with various elongated flat and high-dome blisters. The orientation of the grains was determined by EBSD and is represented by the inserted cubes. The projected $\{110\}$ slip planes of the slip system $\{110\}<111>$ are partly marked. The surface was tilted by $70^{\circ}$ and the image was stretched in vertical direction in order to correct the tilt shortening. 


\section{References}

[1] Roth J et al. 2008 Plasma Phys. Control. Fusion 50103001

[2] Causey RA, Venhaus T J 2001 Physica Scripta T94 9

[3] Causey RA 2002 J. Nucl. Mater. 30091

[4] Skinner CH et al. 2008 Fusion Sci. Technol. 54891.

[5] Haasz AA, Poon M, Davis J 1999 J. Nucl. Mater. 266-269 520

[6] Tokunaga $\mathrm{K}$ et al. $2005 \mathrm{~J}$. Nucl. Mater 337-339 887

[7] Shu WM, Wakai E and Yamanishi T 2007 Nucl. Fusion 47201

[8] Shu WM 2008 Appl. Phys. Letters 92211904

[9] Shu WM, Kawasuso A, Yamanishi T 2009 J. Nucl. Mater 386-388 356

[10] Shu WM et al. 2009 J. Nucl. Mater 390-391 1017

[11] Shu WM, Luo G-N, Yamanishi T 2007 J. Nucl. Mater 367-370 1463

[12] Alimov VKh et al. 2008 J. Nucl. Mater. 375192

[13] Scherzer BMU, in: Behrisch R (Ed.), Sputtering by Particle Bombardment II (Berlin: Springer, 1983) pp. 271-355.

[14] Haasz AA et al. 1998 J. Nucl. Mater 258-263 889

[15] Ueda Y, Shimada T, Nishikava M 2004 Nucl. Fusion 4462

[16] Nishijima D, et al 2005 Nucl. Fusion 45669

[17] Fukumoto M, et al 2008 J.Nucl.Mater. 375224

[18] Enomoto N, et al 2009 J. Nucl. Mater. 385606

[19] Randel RF, Kulcinski GL 2007 J. Nucl. Mater. 367-370 434

[20] Fu Z, et al 2004 J. Nucl. Mater. 329-330 692

[21] Cipiti BB, Kulcinski GL 2005 J. Nucl. Mater. 347298

[22] Alimov VKh et al., Surface morphology and deuterium retention in tungsten exposed to lowenergy, high-flux pure and helium-seeded deuterium plasmas, these proceedings, submitted to Physica Scripta

[23] Alimov VKh et al., Temperature dependence of surface topography and deuterium retention in tungsten exposed to low-energy, high-flux D plasma, proceedings of ICFRM-14, Sapporo, Sep. 2009, submitted to J. Nucl. Mater

[24] Causey RA et al. 1999 J. Nucl. Mater 266-269 467

[25] Luo G-N, Shu W M, Nishi M, 2006 Fusion Engineering and Design 81957

[26] Luo G-N et al. 2004 Rev. Sci. Instrum. 754374

[27] Eleveld H, van Veen A 1992 J. Nucl. Mater. 191-194 433

[28] Van Veen A et al 1988 J. Nucl. Mater. 155-157 1113

[29] Gumbsch P et al. 1998 Science 2821293

[30] Giannattasio A et al. 2007 Physica Scripta T128 87

[31] Lassner E and Schubert WD 1999 Tungsten (New York: Kluwer Academic)

[32] Yalcinkaya1 T, Brekelmans WAM and Geers MGD, Modelling Simul. Mater. Sci. Eng. 16 (2008) 085007

[33] Franzen P et al 1997 J. Nucl. Mater. 241-243 1082

[34] Sakamoto R et al 1996 J. Nucl. Mater. 233-237 776 